Article

\title{
Analytical Analysis of a Novel Brushless Hybrid Excited Adjustable Speed Eddy Current Coupling
}

\author{
Yibo Li ${ }^{1}{ }^{(}$, , Heyun Lin ${ }^{1, *}$, Hai Huang ${ }^{2}$, Hui Yang ${ }^{1}{ }^{(0)}$, Qiancheng Tao ${ }^{1}$ and Shuhua Fang ${ }^{1}$ \\ 1 School of Electrical Engineering, Southeast University, Nanjing 210096, China; epolee@163.com (Y.L.); \\ huiyang@seu.edu.cn (H.Y.); 220162293@seu.edu.cn (Q.T.); shfang@seu.edu.cn (S.F.) \\ 2 Jiangsu Magnet Valley Technologies Co., Ltd., Zhenjiang 212009, China; 13871110369@163.com \\ * Correspondence: hyling@seu.edu.cn; Tel.: +86-025-8379-4169 (ext. 805)
}

Received: 19 December 2018; Accepted: 17 January 2019; Published: 19 January 2019

\begin{abstract}
A novel brushless hybrid excited adjustable speed eddy current coupling is proposed for saving energy in the drive systems of pumps and fans. The topology and operation principle of the coupling are presented. Based on the real flux paths, the fluxes excited by permanent magnet (PM) and field current are analyzed separately. A magnetic circuit equivalent (MEC) model is established to efficiently compute the no-load magnetic field of the coupling. The eddy current and torque are calculated based on the proposed MEC model, Faraday's law, and Ampere's law. The resultant magnetic fields, eddy currents, and torques versus slip speeds under different field currents are studied by the MEC-based analytical method and verified by finite element analysis (FEA). The copper loss, core loss, and efficiency were investigated by FEA. The analytically predicted results agree well with the FEA, and the analysis results illustrate that a good speed regulation performance can be achieved by the proposed hybrid excited control.
\end{abstract}

Keywords: eddy current coupling; hybrid excited; magnetic equivalent circuit; magnetic field analysis; torque-slip characteristic

\section{Introduction}

The eddy current coupling forms a basic variable speed drive, which is widely applied to regulate the flows from large pumps and fans for a remarkable energy-saving effect [1]. With the development of permanent magnet (PM) material, a high torque density adjustable speed PM eddy current coupling (AS-PMECC) (Oregon State University, Corvallis, OR, USA) has been developed and applied in the field of variable speed drive [2]. Compared with other adjustable speed devices, such as gearboxes [3] and variable frequency drives [4], AS-PMECC has many advantages, such as lower sensitivity to environmental conditions, more reliable overload protection, and better energy-saving performance $[5,6]$.

Based on the magnetized directions of PMs, AS-PMECC can be generally classified as axial flux [7] and radial flux couplings [8]. It is usually installed between prime motor and load, which commonly consists of a PM rotor (PMR), a conductor rotor (CR), and an additional mechanical manipulator (AMM) [6]. When a relative rotation happens between the two rotors, eddy currents can be induced in the copper sheet (CS) mounted on the iron core of the CR, which yields an electromagnetic torque from the interaction with the primary PM magnetic field [9-12]. For a given load torque, the speed of AS-PMECC can be adjusted by changing either the air-gap length or coupling area between the two rotors by the AMM $[6,10]$.

In essence, the mechanical-based solutions usually realize speed regulation by adjusting the air-gap flux between the PMR and the CR. However, it is relatively hard to regulate the axial displacement between the two rotors, which requires a relative complicated design of AMM. Further, 
the complicated AMM is not reliable enough and takes more axial space, which is urgent to be simplified and improved for the energy-saving reconstruction of aged pump and fan systems. In addition, the local overheat problem produced by shifting a rotor is very serious in the conventional radial flux AS-PMECC $[8,13]$. Therefore, this paper proposes a novel brushless hybrid excited adjustable speed eddy current coupling (HE-ASECC) (Southeast University, Nanjing, China ) based on a hybrid excited concept to solve the above problem [14,15].

The novelty of the proposed coupling lies on the brushless hybrid excited (HE) control by an additional field excitation stator (AFES), which can provide a flexible air-gap field adjustment. The AFES just occupies some radial space rather than axial space, which is very suitable for the energy-saving reconstruction of aged pump and fan systems. Due to the absence of brush, slip ring, and complicated AMM, the magnetic field control of the HE-ASECC becomes highly simple and reliable. For illustrating the advantages of the HE-ASECC more clearly, it is compared with valve and baffling vane (VB), AS-PMECC, and variable frequency drive (VFD) in Appendix A. Due to the special geometry of the coupling, three-dimensional (3D) finite element analysis (FEA) is usually required to accurately compute its electromagnetic characteristics, but it is very time-consuming. For improving computational efficiency, an analytical model based on the magnetic equivalent circuit (MEC) method is proposed to analyze the electromagnetic characteristics of the coupling. The eddy current and torque are calculated and analyzed based on Ampere's laws under an asymmetric magnetic field. Finally, the analytically predicted results are presented and compared with the FEA.

\section{Structure and Principle}

Figure 1a shows the structure of the studied HE-ASECC. It consists of an AFES, a PMR, and a CR, all of which are coaxial. Different from the AMM used in the conventional AS-PMECC, the AFES embedded with a toroidal field winding is located outside the PMR. Usually, the PMR and the CR are connected with the load mover and the prime motor, respectively. The PMR is characterized by two suits of axially parallel consequent-poles mounted on the inner surface of the PMR core that consists of two annular iron cores fixed together with an axial distance. The PMs mounted on the two annular iron cores are radial magnetized in opposite directions, respectively. The copper sheet (CS) is tightly mounted on the surface of iron core of the $\mathrm{CR}$ to provide paths for the induced eddy currents.

The field control principle can be explained by the magnetic fluxes passing through the PM and iron poles as shown in Figure 1b-d under different field currents. The PMs serve as constant magnetomotive force (MMF) sources, while the field winding acts as a changeable MMF source to perform a flexible air-gap flux adjustment. As a result, the fluxes produced by the field current can enhance or weaken the air-gap fluxes produced by PMs alone based on the directions and amplitudes of the applied field currents. Consequently, the slip speed between the PMR and the CR under a given load torque can be adjusted with the aid from HE control.

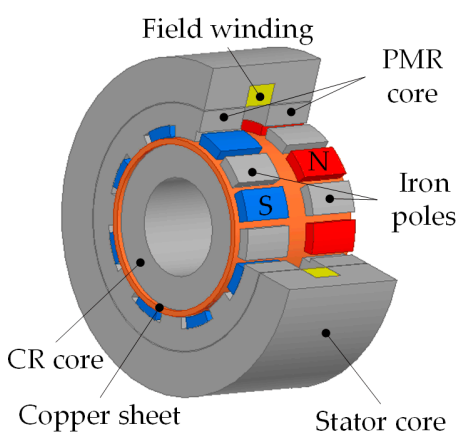

(a)

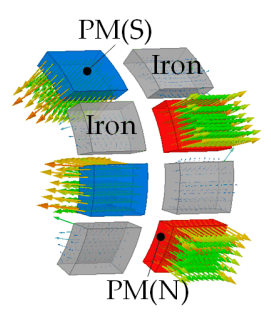

(b)

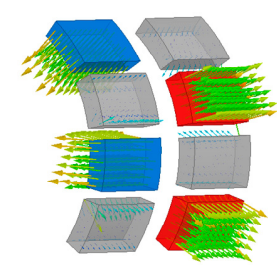

(c)

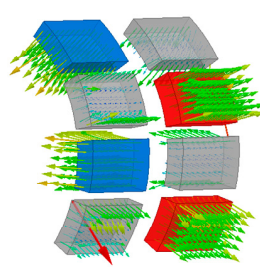

(d)

Figure 1. The magnetic field control principle of the coupling. (a) The topology. (b) $I_{f}=-10 \mathrm{~A}$. (c) $I_{f}=$ 0 A. (d) $I_{f}=10$ A. 


\section{Analytical Model}

In the design process of an electromagnetic device, a reliable and effective theoretical model is desirable for evaluating its electromagnetic characteristics. Generally, the theoretical models based on the magnetic vector potential $[9,16]$ and the MEC method [17-21] are very popular for the design and analysis of electromagnetic devices. Considering the special 3D structure of the proposed HE-ASECC, the latter is adopted in this paper.

\subsection{No-Load Magnetic Field Calculation}

Based on the real fluxes shown in Figure $1 \mathrm{~b}-\mathrm{d}$ and the reluctance elements of MEC, the fluxes created by the PMs and the field current can be analyzed separately. In addition, it should be noted that the CR core and the stator core are communal for the MEC branches excited by the PMs and the field current. Figure $2 \mathrm{a}, \mathrm{b}$ give the MEC models of the coupling excited by both the PMs and the field current when the slip speed between the two rotors is $0 \mathrm{rpm}$, respectively.

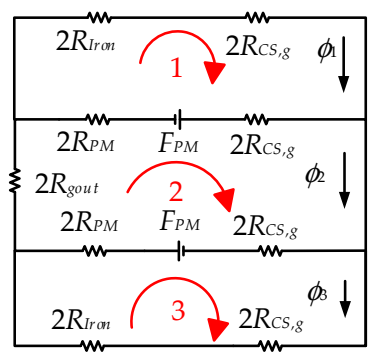

(a)

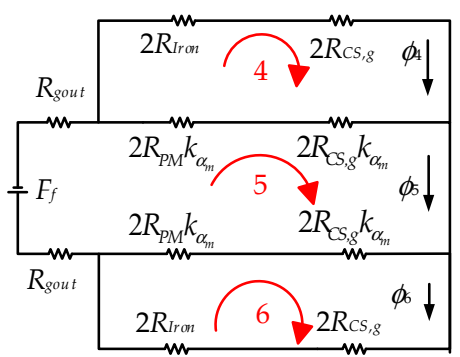

(b)

Figure 2. The magnetic circuit equivalent (MEC) model of the hybrid excited adjustable speed eddy current coupling (HE-ASECC). (a) permanent magnet (PM) excited. (b) If excited.

As shown in Figure 2a, the PM fluxes can be divided into two parts. One passes through the adjacent PM poles located on the two annular iron cores, the inner and outer air-gaps and the CS, and loops with the CR core and the stator core. The other begins with the PM poles and passes through the adjacent iron poles located on the same annular iron core, the inner air-gap and the CS, and loops with the PMR core and the CR core. As shown in Figure $2 b$, the fluxes created by the field winding pass through the outer air-gap, the PMR cores, the iron pole, and the PM-air region, including the PM poles and the air region between PM and iron poles located on the same annular iron core, the inner air-gap and the CS, and loop with the CR core. All elements of the MEC model are calculated in the cylindrical coordinate to permit higher accuracy. In addition, the symbols describing the structure parameters of the coupling are presented in its axial section and axial cross section shown in Figure 3a,b, respectively.

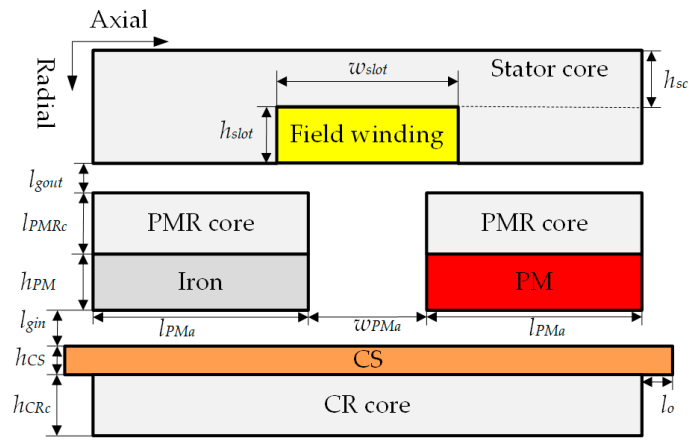

(a)

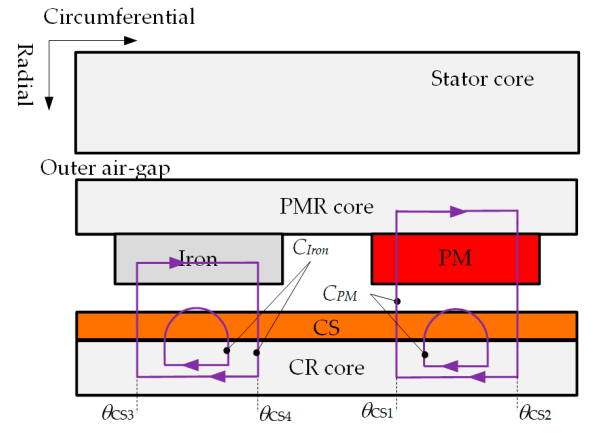

(b)

Figure 3. The 2D view of the HE-ASECC. (a) Axial section. (b) Axial cross section. 
Assuming the iron parts of the coupling are unsaturated, the reluctances of the stator core, the PMR core, the iron poles, and the CR core can be ignored for simplifying calculation. The MMF sources are constituted by the PM poles and the field winding located in the AFES, which can be respectively expressed as

$$
\begin{gathered}
F_{P M}=H_{c} h_{P M} \\
F_{f}=I_{f} N_{f}
\end{gathered}
$$

where $I_{f}, N_{f}, H_{c}$, and $h_{P M}$ represent the field current, the turns of the field winding, the PM coercive force, and the thicknesses of the PM poles, respectively. Applying Kirchhoff's voltage (KVL) law to Loops $1-6$ of the MEC model shown in Figure 2 yields $[17,18]$

$$
\begin{aligned}
& {\left[\begin{array}{ccc}
R_{11} & -R_{12} & 0 \\
-R_{21} & R_{22} & -R_{23} \\
0 & -R_{32} & R_{33}
\end{array}\right] \times\left[\begin{array}{l}
\phi_{1} \\
\phi_{2} \\
\phi_{3}
\end{array}\right]=\left[\begin{array}{c}
-F_{P M} \\
2 F_{P M} \\
-F_{P M}
\end{array}\right]} \\
& {\left[\begin{array}{ccc}
R_{44} & -R_{45} & 0 \\
-R_{54} & R_{55} & -R_{56} \\
0 & -R_{65} & R_{66}
\end{array}\right] \times\left[\begin{array}{c}
\phi_{4} \\
\phi_{5} \\
\phi_{6}
\end{array}\right]=\left[\begin{array}{c}
0 \\
-F_{f} \\
0
\end{array}\right]}
\end{aligned}
$$

where $\phi_{1}$ to $\phi_{6}$ are the fluxes flowing along Loops 1-6 in the MEC model.

$$
\begin{gathered}
\left\{\begin{array}{l}
R_{11}=R_{33}=2\left(R_{P M}+2 R_{C S, g}+R_{\text {Iron }}\right) \\
R_{22}=4\left(R_{P M}+R_{C S, g}+R_{\text {gout }}\right) \\
R_{12}=R_{21}=R_{23}=R_{32}=2\left(R_{P M}+R_{C S, g}\right)
\end{array}\right. \\
\left\{\begin{array}{l}
R_{44}=R_{66}=2\left(k_{\alpha_{m}} R_{P M}+2 k_{\alpha_{m}} R_{C S, g}+R_{\text {Iron }}\right) \\
R_{55}=4\left(k_{\alpha_{m}} R_{P M}+k_{\alpha_{m}} R_{C S, g}+R_{\text {gout }}\right) \\
R_{45}=R_{54}=R_{56}=R_{65}=2\left(k_{\alpha_{m}} R_{P M}+k_{\alpha_{m}} R_{C S, g}\right)
\end{array}\right.
\end{gathered}
$$

where $R_{P M}, R_{\text {Iron }}, R_{C S, g}, R_{\text {gout }}$, and $k_{\alpha m}$ denote the reluctances of the PM pole, the iron pole, the copper sheet and the inner air-gap, the outer air-gap, and the coefficient to modify $R_{P M}$ and $R_{C S, g}$ under the field current excitation alone, respectively. Assuming the permeabilities of the CS and PM are equal to $\mu_{0}$, the above parameters can be calculated as $[17,18]$

$$
\left\{\begin{array}{l}
R_{P M}=\ln \left[1+h_{P M} /\left(r_{C S o}+l_{\text {gin }}\right)\right] /\left(\mu_{0} \alpha_{m} \tau_{p} l_{P M a}\right) \\
R_{C S}=\ln \left(1+h_{C S} / r_{C S i}\right) /\left(\mu_{0} \alpha_{m} \tau_{p} l_{P M a}\right) \\
R_{\text {gin }}=\ln \left(1+l_{\text {gin }} / r_{C S o}\right) /\left(\mu_{0} \alpha_{m} \tau_{p} l_{P M a}\right) \\
R_{\text {gout }}=2 \ln \left(1+l_{\text {gout }} / r_{P M R c o}\right) /\left[\mu_{0} \alpha_{m} \tau_{p}\left(2 l_{P M a}+w_{P M a}-w_{\text {slot }}\right)\right] \\
R_{C S, g}=R_{C S}+R_{\text {gin }} \\
k_{\alpha_{m}}=\left(2-\alpha_{m}\right) / \alpha_{m}
\end{array}\right.
$$

where $r_{C S i}, r_{C S o}, r_{P M R c o}, \alpha_{m}, \tau_{p}, l_{P M a}$, and $\mu_{0}$ is the inner and outer radii of the CS, the outer radius of the PMR core, the pole-arc coefficients of PM and iron poles, the pole pitch in the circumferential direction, the axial length of PM poles, and the vacuum permeability, respectively.

Based on the analysis of the flux paths and reluctance parameters of the coupling, the flux density distributions can be obtained by a superposition of the fields produced by the PMs and the field current. Based on the MEC model in Figure 2 and the flux density model in Figure 4, the no-load air-gap flux density of the coupling can be expressed as $[17,18,20]$

$$
B_{P M}(\theta)=\left\{\begin{array}{lr}
k_{t} B_{P M \_} P M\left(\theta-\theta_{2}\right) /\left(\theta_{2}-\theta_{1}\right)+B_{P M_{-} I_{f}} ; \theta_{1} \leq \theta<\theta_{2} \\
k_{t} B_{P M \_}+\theta_{P M}+B_{P I_{-} I_{f}} ; & \theta_{2} \leq \theta 3 \\
k_{t} B_{P M_{-} P M}\left(\theta-\theta_{4}\right) /\left(\theta_{3}-\theta_{4}\right)+B_{P M_{-} I_{f}} ; \theta_{3}<\theta \leq \theta_{4}
\end{array}\right.
$$




$$
B_{\text {Iron }}(\theta)=\left\{\begin{array}{lr}
k_{t} B_{\text {Iron_PM }}\left(\theta-\theta_{2}\right) /\left(\theta_{2}-\theta_{1}\right)+B_{P M_{-} I_{f}} ; \theta_{1} \leq \theta<\theta_{2} \\
k_{t} B_{\text {Iron_PM }}+B_{\text {Iron_I }} ; & \theta_{2} \leq \theta \leq \theta_{3} \\
k_{t} B_{\text {Iron_PM }}\left(\theta-\theta_{4}\right) /\left(\theta_{3}-\theta_{4}\right)+B_{P M_{-} I_{f}} ; \theta_{3}<\theta \leq \theta_{4}
\end{array}\right.
$$

where $k_{t}$ denotes the coefficient to modify the trapezoid flux density waveforms shown in Figure 4a,b. $\theta_{1}$ to $\theta_{4}$ and $k_{t}$ can be obtained as follows:

$$
\left\{\begin{array}{l}
\theta_{1}=-\theta_{4}=-\tau_{p} / 2 \\
\theta_{2}=-\theta_{3}=-\tau_{p} \alpha_{m} / 2 \\
k_{t}=2 \alpha_{m} /\left(1+\alpha_{m}\right)
\end{array}\right.
$$

In addition, $B_{P M_{-} P M}, B_{I r o n \_P M}, B_{P M \_I f}$, and $B_{\text {Iron_If }}$ denote the average flux densities facing the PM and iron poles produced by the PM and the field current, respectively, which can be derived as

$$
\left\{\begin{array}{l}
B_{P M_{-} P M}=2\left(\phi_{2}-\phi_{1}\right) /\left[\tau_{p} \alpha_{m}\left(r_{C S o}+l_{g} / 2\right) l_{P M}\right] \\
B_{I r o n \_P M}=2 \phi_{1} /\left[\tau_{p} \alpha_{m}\left(r_{C S o}+l_{g} / 2\right) l_{P M}\right] \\
B_{P M_{-} I_{f}}=2\left(\phi_{5}-\phi_{4}\right) /\left[\tau_{p}\left(2-\alpha_{m}\right)\left(r_{C S o}+l_{g} / 2\right) l_{P M}\right] \\
B_{I r n_{-} I_{f}}=2 \phi_{4} /\left[\tau_{p} \alpha_{m}\left(r_{C S o}+l_{g} / 2\right) l_{P M}\right]
\end{array}\right.
$$

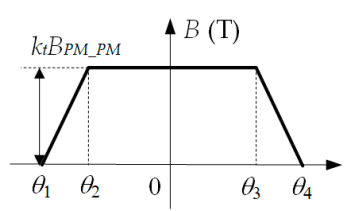

(a)

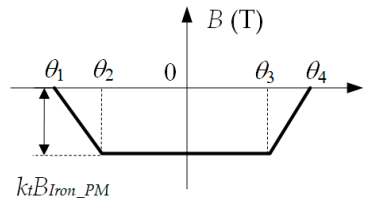

(b)

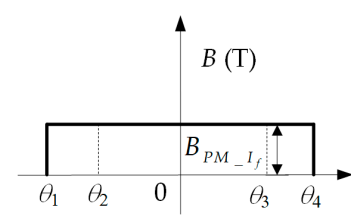

(c)

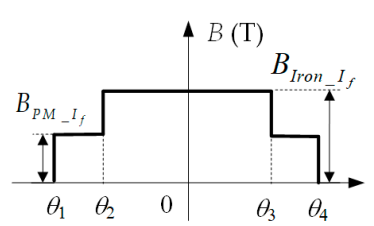

(d)

Figure 4. The model of air-gap flux density. (a) $B_{P M}$ excited by PMs. (b) $B_{I r o n}$ excited by PMs. (c) $B_{P M}$ excited by $I_{f}$. (d) $B_{\text {Iron }}$ excited by $I_{f}$.

\subsection{Eddy Current Field Calculation}

Thanks to FEA, the distributions of eddy currents under different field currents can be easily calculated and shown in Figure 5 when the slip speed is $150 \mathrm{rpm}$. It can be seen that the directions of eddy currents do not change, while the direction of the field current is reversed. In addition, the eddy current densities rise with the increase in field current.

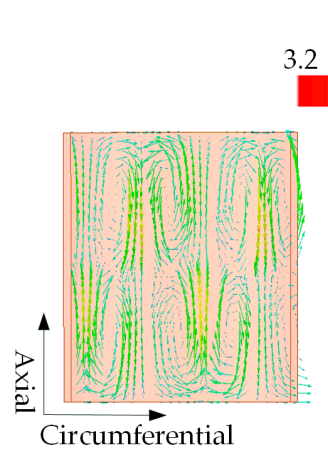

(a)

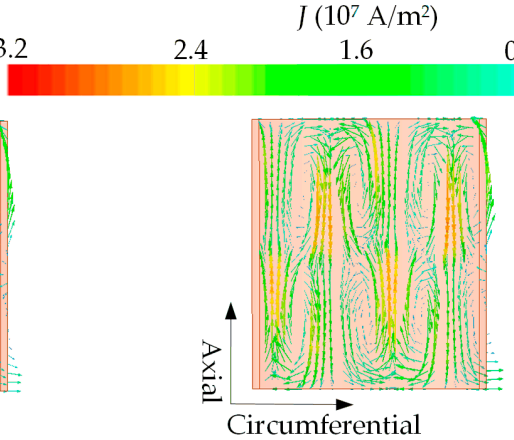

(b)

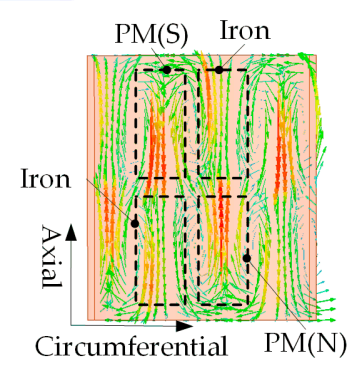

(c)

Figure 5. The distributions of eddy currents. (a) $I_{f}=-10 \mathrm{~A}$. (b) $I_{f}=0 \mathrm{~A}$. (c) $I_{f}=10 \mathrm{~A}$. 
As a relative rotation between the PMR and the CR happens, the eddy current will be induced in the CS. Since the air-gap flux densities facing the PM and iron poles are different, the eddy current densities can be calculated separately, which can be respectively given as $[17,18,20]$

$$
\begin{aligned}
& J_{P M}(r, \theta)=r \sigma \omega B_{r_{-} P M}(\theta) \\
& J_{\text {Iron }}(r, \theta)=r \sigma \omega B_{r_{-} I r o n}(\theta)
\end{aligned}
$$

where $\sigma$ and $\omega$ denote the conductivity of the CS and relative angular velocity, respectively. $B_{r_{-} P M}(\theta)$ and $B_{r_{-} \text {Iron }}(\theta)$ are the radial components of the resultant flux density in the inner air-gap facing the PM and iron poles, respectively. In fact, the axial components of the eddy current densities facing the PM and iron poles in the same loop are the same, i.e., the average eddy current densities of $J_{P M}$ and $J_{I r o n}$ are equal, which can be expressed as

$$
J_{a v}(r, \theta)=\left(J_{P M}(r, \theta)-J_{I r o n}(r, \theta)\right) / 2=r \sigma \omega\left(B_{r_{-} P M}(\theta)-B_{r_{-} I r o n}(\theta)\right) / 2 .
$$

In fact, the PM magnetic field is interacted by the magnetic field produced by the eddy current in the CS. Thus, an iterative process should be taken in the air-gap field calculation of the coupling. The air-gap flux density is first determined using the no-load MEC model. The eddy current density in the CS is then calculated by means of Faraday's law under the different slip speeds between the two rotors. Afterward, the impact of the magnetic field yielded by the eddy currents on the original air-gap flux density is taken into account through Ampere's law. The radial components of the resultant flux densities in the air-gap can be expressed as $[17,18,20]$

$$
\begin{aligned}
& B_{r_{-} P M}^{(n)}(\theta)=B_{P M}(\theta)+B_{C S_{-} P M}^{(n-1)}(\theta) \\
& B_{r_{-} I r o n}^{(n)}(\theta)=B_{\text {Iron }}(\theta)+B_{C S_{-} \text {Iron }}^{(n-1)}(\theta)
\end{aligned}
$$

where the positive integer $n$ represents the iteration number, and $B_{C S_{-} P M}(\theta)$ and $B_{C S_{-} I r o n}(\theta)$ denote the flux densities produced by the eddy current in the CS facing the PM and iron poles, respectively. The eddy current field can be calculated based on Ampere's laws expressed as $[17,18,20]$

$$
\begin{aligned}
\int_{C_{P M}} H d l & =\int_{\theta_{C S 1}}^{\theta_{C S 2}} \int_{r_{C S i}}^{r_{C S O}} J_{P M}(r, \theta) r d r d \theta \\
\int_{C_{\text {Iron }}} H d l & =\int_{\theta_{C S 3}}^{\theta_{C S 4}} \int_{r_{C S i}}^{r_{C S O}} J_{\text {Iron }}(r, \theta) r d r d \theta
\end{aligned}
$$

where the right terms are the total currents enclosed in flux paths $C_{P M}$ and $C_{I r o n} . \theta_{C S 1}$ to $\theta_{C S 4}$ are the edges of the eddy current field flux paths shown in Figure $3 b$.

Based on Equations (17) and (18), the eddy currents under the iron and PM poles are integrated along the different lengths of the flux paths $\left(C_{P M}\right.$ and $\left.C_{\text {Iron }}\right)$ shown in Figure $3 \mathrm{~b}$. For simplifying calculation, the magnetic reluctances of the iron materials are ignored. Thus, the average lengths of the flux paths facing the PM and iron poles can be expressed as $l_{e_{-} P M}=\pi\left(\tau_{p} / 4\right)$ and $l_{e_{-} \text {Iron }}=2\left(l_{g i n}+h_{C S}\right)$, respectively [20]. Based on Equations (14), (17), and (18) and the selections of paths $C_{P M}$ and $C_{\text {Iron }}$ in Figure $3 \mathbf{b}$, the flux densities $B_{C S_{-} I r o n}(\theta)$ and $B_{C S_{-} P M}(\theta)$ can be respectively obtained as $[17,18,20]$

$$
\begin{gathered}
B_{C S \_ \text {Iron }}=-k_{l e} B_{C S_{-} \text {Iron }} \\
B_{C S_{-} P M}(\theta)=\left\{\begin{array}{lr}
B_{C S 1}=k_{1} e^{m^{\prime} \theta} ; & \theta_{1} \leq \theta<\theta_{2} \\
B_{C S 2}=k_{2} e^{m^{\prime} \theta}-B_{m} /\left(1+k_{l e}\right) & \theta_{2} \leq \theta \leq \theta_{3} \\
B_{C S 3}=k_{3} e^{m^{\prime} \theta} ; & \theta_{3}<\theta \leq \theta_{4}
\end{array}\right.
\end{gathered}
$$


where

$$
\begin{aligned}
& B_{m}=\left(B_{P M \_} P M+B_{P M_{-} I_{f}}-B_{I r o n_{-} P M}-B_{I_{\text {Iron }} I_{f}}\right) / 2 \\
& m^{\prime}=m_{P M}\left(1+k_{l e}\right) / 2 \\
& k_{l e}=l_{e_{\_} P M} / l_{e_{-} I r o n} \\
& m_{P M}=\mu_{0} \sigma \omega\left(r_{C S o}^{3}-r_{C S i}^{3}\right) /\left(3 l_{e_{-} P M}\right)
\end{aligned}
$$

The following boundary conditions are taken as

$$
\begin{gathered}
B_{C S 2}\left(\theta_{0}\right)=0 \\
B_{C S 1}\left(\theta=\theta_{2}\right)=B_{C S 2}\left(\theta=\theta_{2}\right) \\
B_{C S 2}\left(\theta=\theta_{3}\right)=B_{C S 3}\left(\theta=\theta_{3}\right)
\end{gathered}
$$

where Equation (22) is the main boundary condition of the problem referring to a particular point where the total currents enclosed in the intervals $\left[\theta_{1}, \theta_{0}\right]$ and $\left[\theta_{0}, \theta_{4}\right]$ are equal, and Equations (23) and (24) express the continuity of $B_{C S}(\theta)$. $\theta_{0}$ can be determined by

$$
\int_{\theta_{1}}^{\theta_{0}} \int_{r_{C S i}}^{r_{C S o}} J(r, \theta) r d r d \theta=\int_{\theta_{0}}^{\theta_{4}} \int_{r_{C S i}}^{r_{C S o}} J(r, \theta) r d r d \theta
$$

Thus,

$$
\theta_{0}=-\frac{1}{m^{\prime}} \ln \left\{\frac{1+\left(1-e^{\alpha_{m}}\right) \cosh \left[\left(1-\alpha_{m}\right) m^{\prime} \tau_{p} / 2\right]}{\left(1-e^{\alpha_{m}}\right) \cosh \left(m^{\prime} \tau_{p} / 2\right)+\cosh \left(m^{\prime} \alpha_{m} \tau_{p} / 2\right)}\right\} .
$$

The coefficients $k_{1}, k_{2}$ and $k_{3}$ in $(20)$ can then be expressed as $[17,18]$

$$
\left\{\begin{array}{l}
k_{1}=B_{m}\left(e^{-m^{\prime} \theta_{0}}-e^{-\alpha_{m} m^{\prime} \theta_{1}}\right) /\left(1+k_{l e}\right) \\
k_{2}=B_{m} e^{-m^{\prime} \theta_{0}} /\left(1+k_{l e}\right) \\
k_{3}=B_{m}\left(e^{-m^{\prime} \theta_{0}}-e^{-\alpha_{m} m^{\prime} \theta_{4}}\right) /\left(1+k_{l e}\right)
\end{array} .\right.
$$

Thus, the radial components of the air-gap flux densities can be obtained by substituting Equations (19) and (20) into Equations (15) and (16), respectively.

\subsection{Torque Calculation}

The torque of the coupling can be finally determined by using the total ohmic losses dissipated in the CS [17-21]:

$$
T=\frac{K_{s} p_{c u \_c s}}{\omega}=\frac{2 K_{S} l_{P M a}}{\sigma \omega} \iint_{C S}|J(r, \theta)|^{2} r d r d \theta=2 K_{s} l_{P M a} \pi\left(r_{C S o}^{2}-r_{C S i}^{2}\right) J_{a v}^{2} / \sigma \omega
$$

where $K_{s}, p_{c u_{-} C S}$, and $J_{a v}$ denote the 3D coefficient, the copper loss in the CS, and the average eddy current density in the same loop. $K_{S}$ can be derived as [22]

$$
K_{s}=1-\frac{\tanh \left[p l_{P M a} /\left(2 r_{a v}\right)\right] /\left[p l_{P M a} /\left(2 r_{a v}\right)\right]}{1+\tanh \left[p l_{P M a} /\left(2 r_{a v}\right)\right] \tanh \left[p l_{o} /\left(2 r_{a v}\right)\right]}
$$

where $r_{a v}=\left(r_{C S o}+r_{C S i}\right) / 2$, and $l_{0}$ and $p$ denote the overhang-length of the CS and the pole pair numbers of the PMR, respectively.

\subsection{Efficiency}

The output and input powers of the coupling can be respectively expressed as

$$
P_{\text {out }}=T_{\text {out }} \Omega_{\text {out }}
$$




$$
P_{\text {in }}=P_{\text {out }}+p_{c u_{-} C S}+p_{c u_{-} f w}+p_{\text {other }}
$$

where $T_{\text {out }}, \Omega_{\text {out }}$, and $p_{c u f f}$ denote the output torque, the output angular speed, and the copper loss of the field winding, respectively. $p_{\text {other }}$ denotes the other losses including core loss, stray loss, mechanical loss, and so on. The coupling efficiency can then be obtained as

$$
\eta_{C}=P_{\text {out }} / P_{\text {in }} \times 100 \% .
$$

Ignoring other losses, the input torque is almost equal to the output torque. Thus, the input power of the coupling can be estimated as [20]

$$
P_{\text {in }}=(1-s)^{2} P_{N}
$$

where $P_{N}$ and $s$ denote the rated power of pump and fan loads, and the slip between the PMR and the $\mathrm{CR}$, respectively. The efficiency of the coupling can be simplified as

$$
\eta_{C}=(1-s) \times 100 \% .
$$

With the increase in slip, $P_{\text {in }}$ declines much faster than $\eta_{c}$. Based on [6], the energy-saving effect of the system can be still more pronounced when compared to the method through throttling valves and baffles.

\section{Results and Discussions}

The major design parameters of the HE-ASECC are tabulated in Table 1. The air-gap magnetic fields, eddy currents, and torque-slip characteristics are calculated by the proposed analytical method and verified by FEA. The loss and efficiency are subsequently investigated by FEA.

Table 1. Major design parameters of the coupling.

\begin{tabular}{cccc}
\hline Item & Symbol & Value & Unit \\
\hline Inner radical of CS & $r_{C S i}$ & 46 & $\mathrm{~mm}$ \\
CS thickness & $h_{C S}$ & 3 & $\mathrm{~mm}$ \\
CS conductivity & $\sigma$ & $5.77 \times 10^{7}$ & $\mathrm{~S} / \mathrm{m}$ \\
Over hang length & $l_{0}$ & 5 & $\mathrm{~mm}$ \\
CR yoke thickness & $h_{C R c}$ & 10 & $\mathrm{~mm}$ \\
Air-gap length & $l_{g o u t} / l_{g i n}$ & 1 & $\mathrm{~mm}$ \\
PM inner radical & $r_{m i}$ & 50 & $\mathrm{~mm}$ \\
Magnetic pole height & $h_{P M}$ & 6 & $\mathrm{~mm}$ \\
PM axial length & $l_{P M a}$ & 30 & $\mathrm{~mm}$ \\
PM coercive force & $H_{c}$ & -890 & $\mathrm{kA} / \mathrm{m}$ \\
Pole-arc coefficient & $\alpha_{m}$ & 0.8 & \\
Pole pair numbers of the PMR & $p$ & 16 & \\
PMR yoke thickness & $w_{P M a}$ & 10 & $\mathrm{~mm}$ \\
Stator yoke thickness & $h_{P M R c}$ & 10 & $\mathrm{~mm}$ \\
Stator slot width & $h_{S c}$ & 10 & $\mathrm{~mm}$ \\
Stator slot height & $w_{s l o t}$ & 40 & $\mathrm{~mm}$ \\
Field coil turns number & $h_{s l o t}$ & 10 & $\mathrm{~mm}$ \\
\hline
\end{tabular}

\subsection{Air-Gap Magnetic Field}

Figure 6 shows the radial components of the no-load inner air-gap flux densities between the PMR and the CR under different field currents. When $I_{f}$ increases from -10 to $10 \mathrm{~A}$, the amplitude of $B_{P M}$ decreases from about 0.7 to $0.5 \mathrm{~T}$ (about $30 \%$ ), while $B_{\text {Iron }}$ decreases from 0.2 to $-0.3 \mathrm{~T}$. The variation of $B_{P M}$ is smaller that of $B_{I r o n}$, which can be explained by the fact that the reluctance of PM is much larger than that of iron poles. In addition, the difference between analytical and FEA methods 
is found, which is caused by the flux leakage between adjacent PM and iron poles, which is difficult to be considered in the analytical modeling. The above analysis illustrates a fairly good effect of the proposed approach on air-gap magnetic field regulation.

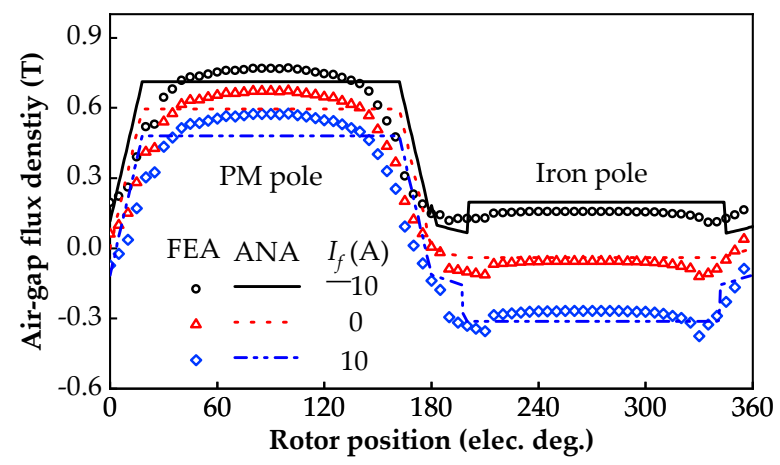

Figure 6. No-load air-gap magnetic flux density distributions.

Figure $7 \mathrm{a}, \mathrm{b}$ show the average values of $B_{r_{-} P M}$ and $B_{r_{-} I r o n}$ as functions of slip speed under different field currents. It is obvious that the average value of $B_{r_{-} P M}$ declines from about 0.62 to $0.41 \mathrm{~T}$, while that of $B_{r_{-} \text {Iron }}$ increases from about -0.3 to $0.15 \mathrm{~T}$ when the slip speed is equal to $50 \mathrm{rpm}$. With the increase in slip speed, the average values of $B_{r_{-} P M}$ and $B_{r_{-} I r o n}$ decline and rise gradually, respectively. Based on Equations (14) and (17), the eddy current field is enhanced with the increase in slip speed. Although the direction of the flux passing through iron pole is changed by the opposite field currents (e.g., $I_{f}=-10$ and $10 \mathrm{~A}$ ), the direction of the eddy current field remains unchanged. The reason is that the direction of eddy current loop is mainly determined by $J_{P M}$, which is much larger than $J_{I r o n}$.

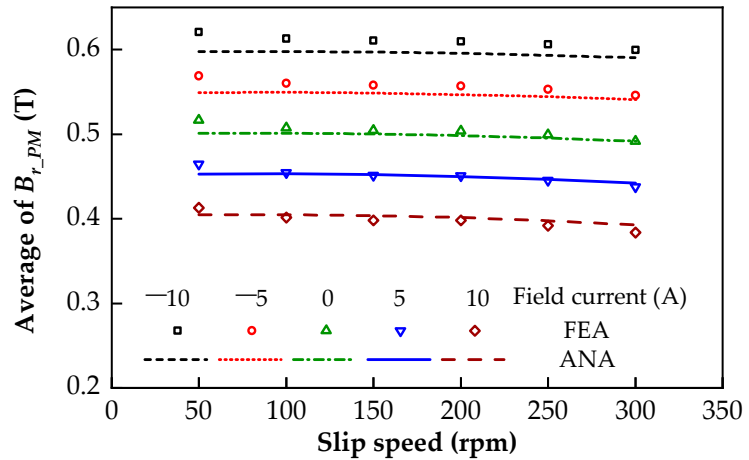

(a)

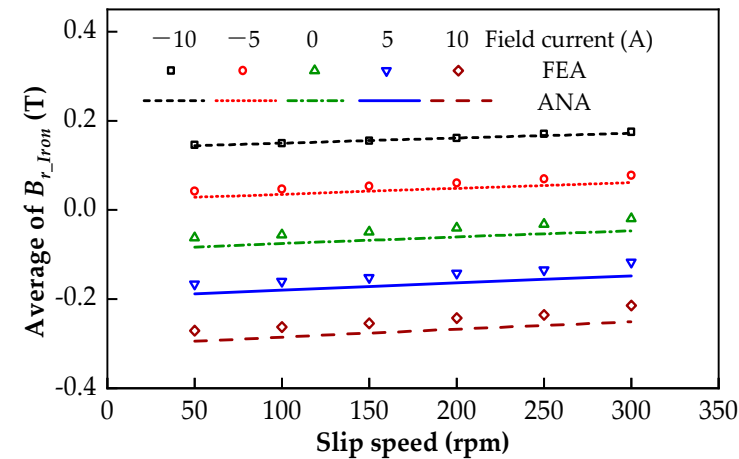

(b)

Figure 7. The average values of flux densities under different field currents. (a) The average values of $B_{r_{-} P M}$. (b) The average values of $B_{r_{-} I r o n}$.

\subsection{Eddy Current Density}

As expected, the eddy current densities facing the PM and iron poles increase gradually with the increase in field current as shown in Figure 8. In addition, the eddy current versus rotor position distributions under the PM and iron poles are not uniform, which can be explained by Equations (12)-(14) and the air-gap flux density distributions shown in Figure 6. It should be noted that the directions of eddy currents facing the iron pole are unchanged when the field current changes from -10 to $10 \mathrm{~A}$. The reason is that $J_{P M}$ and $J_{I r o n}$ are respectively proportional to $B_{r_{-} P M}$ and $B_{r_{-} I r o n}$, while $B_{r_{-} P M}$ is much larger than $B_{r_{-} I r o n}$, which determines the flowing directions of eddy currents.

Figure 9 shows the variations in average eddy current densities facing the PM and iron poles of the proposed coupling under different slip speeds. With the increase in field current, both $J_{P M}$ and $J_{\text {Iron }}$ rise gradually, and they also increase with the increase in slip speed under a given field 
current. It should be noted that the average eddy current densities calculated by analytical and FEA methods agree well, which illustrates that the eddy current calculation model shown in Equation (14) is sufficiently reliable.

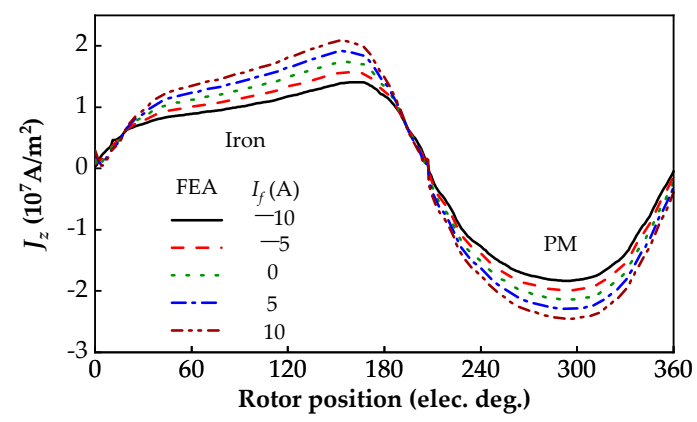

Figure 8. The eddy current densities versus the rotor position.

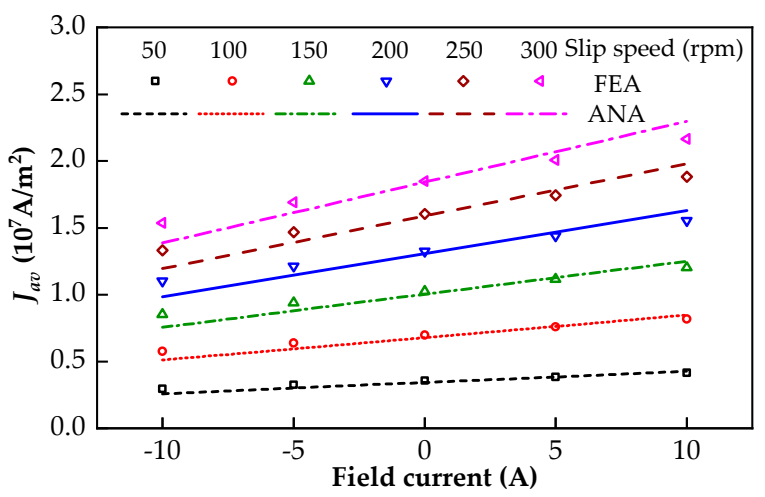

Figure 9. The average eddy current densities under different slip speeds.

\subsection{Torque Characteristics}

Figure 10 shows the torque-slip characteristics of the proposed coupling under different field currents. In the lower slip speed range, the torque presents an ascending trend, the reason of which is that the eddy current density increases proportionally with slip speed. Due to the skin effect, the torque increases more and more slowly. Moreover, it can be seen that the torque experiences a gradually reduction with the decrease in $I_{f}$ from 10 to $-10 \mathrm{~A}$ at a given slip speed. This implies that a wide torque regulation range can be achieved by field control. The above analysis confirms the validity of HE control for the load speed regulation by the proposed coupling.

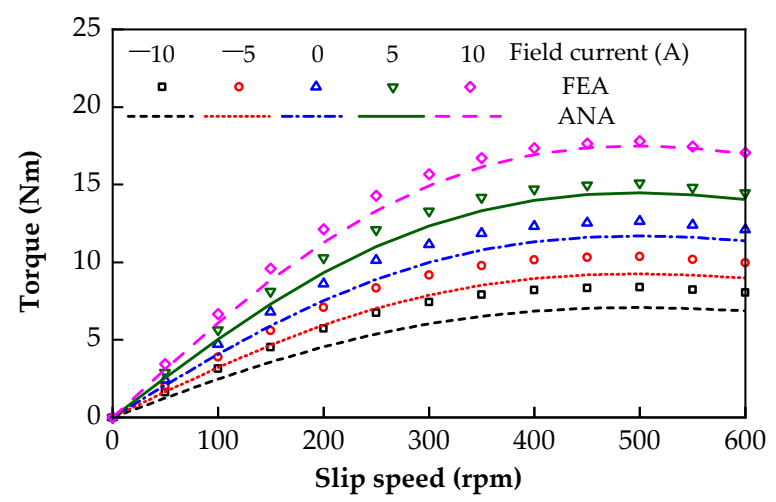

Figure 10. The torque-slip characteristics under different field currents. 


\subsection{Losses and Efficiency}

Figure 11 shows the core losses of the coupling versus slip speed under different field currents. With the increase in slip speed, the core losses of the coupling gradually increase, and are proportional to the frequencies of the alternating magnetic fluxes passing through the cores. In fact, the magnetic fluxes passing through the PMR core and the stator core are almost not alternating, which results in low core losses in the PMR and stator cores. Thus, the core loss is mainly produced in the CR core. In addition, the core loss also increases with the increase in field current, which enhances the alternating magnetic fluxes passing through the CR core.

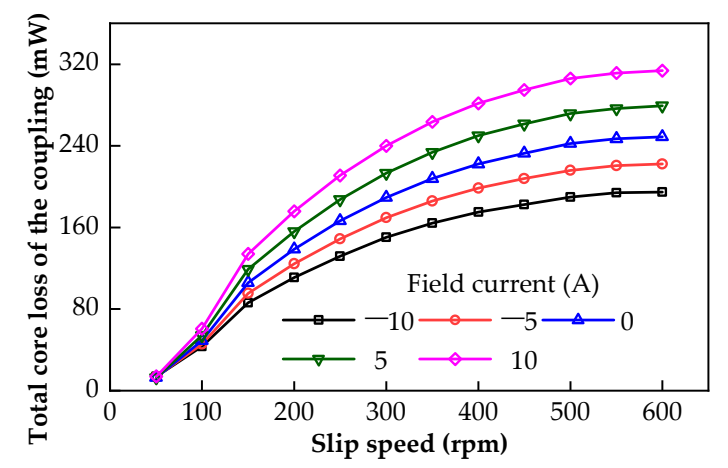

Figure 11. The core losses under different field currents.

Figure 12 shows the copper losses of the CS versus slip speed under different field currents. With the increase in slip speed and field current, the copper losses rise gradually. The copper loss of field winding is proportional to the field current, which can obtain the maximum value as $97.5 \mathrm{~W}$ when the magnitude of the field current is set to $10 \mathrm{~A}$. It takes up a small percentage of the total copper loss of the coupling, which illustrates that the HE control is acceptable for the speed regulation application.

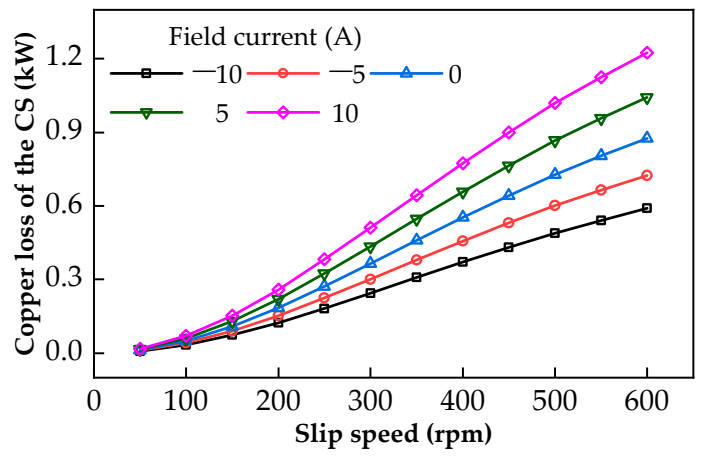

Figure 12. The copper losses of the copper sheet (CS) under different field currents.

Figure 13 shows the efficiencies of the coupling under different field currents when the speed of the prime motor is $1500 \mathrm{rpm}$. The efficiency of the coupling reaches the highest when $I_{f}$ is zero, which is attributed to the fact that the copper loss of the field winding is the lowest. It decreases from 96.7 to $56.9 \%$ when the slip speed increases from 50 to $600 \mathrm{rpm}$. When $I_{f}$ decreases from 10 to $0 \mathrm{~A}$, the efficiency of the coupling slightly declines, while its reduction is enlarged with the further decrease in $I_{f}$ from 0 to $-10 \mathrm{~A}$. This can be attributed to the fact that $P_{\text {out }}$ decreases with the decrease in the field current. In addition, $p_{c u}$ f $w$ declines when the field current decreases from 10 to $0 \mathrm{~A}$, and it then rebounds to a higher value with the further decrease in the field current. In a lower slip speed range, the variations in efficiencies are obvious under different field currents. It is responsible for this that the copper loss of field winding occupies a large percentage of the relatively small output power when the torque of the coupling is small. Although the efficiency of the HE-ASECC declines with the increase in 
slip speed linearly, for a pump system, an energy-saving effect can still be achieved based on Equation (33) compared with the valves and baffles controls.

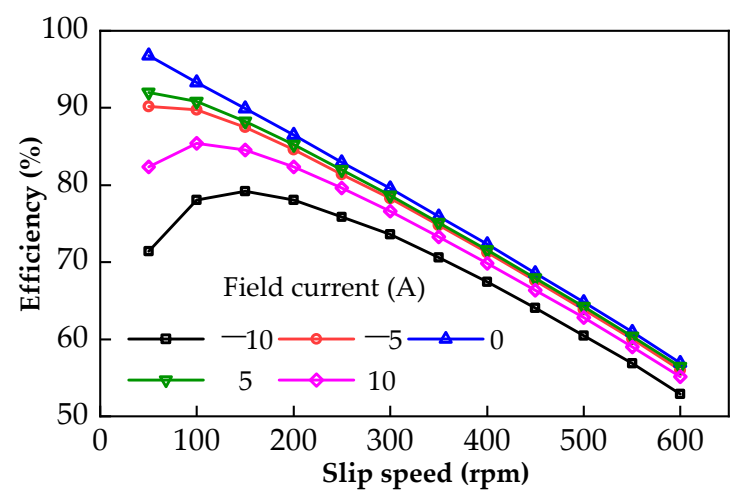

Figure 13. The efficiencies of the coupling under different field currents.

\section{Conclusions}

This paper proposes a novel brushless HE-ASECC with a simple AFES, which makes the online speed regulation reliably and effectively for the energy-saving of the aged pump and fan systems. The MEC-based analytical method is presented to calculate the magnetic field and torque of the proposed coupling efficiently. In addition, the analytical method provides an approach to solve the eddy current field problem under an asymmetric magnetic field of the PMR. Finally, the magnetic field and torque adjustment ability of the coupling illustrates that a wide speed regulation range can be achieved by the HE control. It also reveals that that the proposed speed regulation method can be applied in the field of variable speed drive for energy-saving reconstruction.

Author Contributions: Conceptualization: Y.L., H.L., and H.Y.; methodology: Y.L., Q.T., and H.L; software: Y.L. and Q.T.; validation: H.Y. and H.L.; formal analysis: Y.L. and H.Y.; investigation: Y.L., H.Y., and H.H.; resources: H.L.; data curation: H.L.; writing—original draft preparation: Y.L.; writing—review and editing: H.L., H.Y., and S.F; visualization: Y.L.; supervision: H.L. and H.H.; project administration: H.L.; funding acquisition: H.L.

Funding: This work was supported by the National Natural Science Foundation of China (51577026).

Conflicts of Interest: The authors declare no conflict of interest.

\section{Appendix A}

Table A1 shows a comparison of HE-ASECC with VB, AS-PMECC, and VFD in energy-saving effect, space, efficiency, power electronic converter capacity, reliability, and cost.

Table A1. Full comparison among different energy saving devices.

\begin{tabular}{ccccc}
\hline Item & VB & AS-PMECC & VFD & HE-ASECC \\
\hline Energy-saving effect & $\star$ & $\star \star \star$ & $\star \star \star \star \star$ & $\star \star \star$ \\
Space occupation & $\star \star \star \star \star$ & $\star \star \star$ & $\star \star$ & $\star \star \star \star$ \\
Efficiency & $\star$ & $\star \star \star$ & $\star \star \star \star \star$ & $\star \star \star$ \\
Reliability & None & None & Large & None \\
Cost & $\star \star \star \star \star$ & $\star \star \star$ & $\star \star$ & $\star \star \star \star$ \\
Power electronic converter capacity & $\star \star \star \star \star$ & $\star \star \star \star$ & $\star \star$ & $\star \star \star \star$ \\
\hline
\end{tabular}

\section{References}

1. Bloxham, D.A.; Wright, M.T. Eddy-current coupling as an industrial variable-speed drive. Proc. IEE 1972, 119, 1149-1154. [CrossRef] 
2. Wallace, A.; Wohlgemuth, C.; Lamb, K. A high efficiency, alignment and vibration tolerant, coupler using high energy-product permanent magnet. In Proceedings of the Seventh International Conference on Electrical Machines and Drives, Durham, UK, 11-13 September 1995; pp. 232-236.

3. Abdelaziz, E.A.; Saidur, R.; Mekhilef, S. A review on energy-saving strategies in industrial sector. Renew. Sustain. Energy Rev. 2011, 15, 150-168. [CrossRef]

4. Khalid, N. Efficient energy management: Is variable frequency drives the solution. Procedia Soc. Behav. Sci. 2014, 145, 371-376. [CrossRef]

5. Saidur, R.; Mekhilef, S.; Ali, M.B.; Safari, A.; Mohammed, H.A. Applications of variable speed drive (VSD) in electrical motors energy-savings. Renew. Sustain. Energy Rev. 2012, 16, 543-550. [CrossRef]

6. Wallace, A.; Jouanne, A. Industrial speed control: Are PM couplings an alternative to VFDs? IEEE Trans. Ind. Appl. 2001, 7, 57-63. [CrossRef]

7. Canova, A.; Vusini, B. Design of axial eddy-current couplers. IEEE Trans. Ind. Appl. 2003, 39, 725-733. [CrossRef]

8. Canova, A.; Vusini, B. Mathematic modeling of rotating eddy-current couplers. IEEE Trans. Magn. 2005, 41, 24-35. [CrossRef]

9. Wang, J.; Lin, H.; Fang, S.; Huang, Y. A general analytical model of permanent magnet eddy current couplings. IEEE Trans. Magn. 2014, 50, 1-9. [CrossRef]

10. Lubin, T.; Rezzoug, A. Steady-state and transient performance of axial-field eddy-current coupling. IEEE Trans. Ind. Electron. 2015, 4, 2287-2296. [CrossRef]

11. Wang, J.; Lin, H.; Fang, S. Analytical prediction of torque characteristics of eddy current couplings having a quasi-Halbach magnet structure. IEEE Trans. Magn. 2016, 52, 1-9. [CrossRef]

12. Dai, X.; Cao, J.Y.; Long, Y.J.; Liang, Q.H.; Mo, J.Q.; Wang, S.G. Analytical modeling of an eddy-current adjustable-speed coupling system with a 3-segment Halbach magnet array. Electr. Power Comp. Syst. 2015, 43, 1891-1901. [CrossRef]

13. Zheng, D.; Wang, D.; Li, S.; Zhang, H.; Yu, L.; Li, Z. Electromagnetic-thermal model for improved axial-flux eddy current couplings with combine rectangle-shaped magnets. IEEE Access 2018, 6, 26383-26390. [CrossRef]

14. Yang, H.; Zhu, Z.Q.; Lin, H.; Chu, W.Q. Flux adjustable permanent magnet machines: A technology status review. Proc. CJEE 2016, 2, 14-30.

15. Aydin, M.; Huang, S.; Lipo, T.A. Design, analysis, and control of a hybrid field-controlled axial-flux permanent-magnet motor. IEEE Trans. Ind. Electron. 2010, 57, 78-87. [CrossRef]

16. Lubin, T.; Rezzoug, A. 3-D analytical model for axial-flux adjust speed eddy-durrent couplings and brakes under steady-state conditions. IEEE Trans. Magn. 2015, 51, 1-12. [CrossRef]

17. Mohammadi, S.; Mirsalim, M.; Vaez-Zadeh, S. Nonlinear modeling of eddy-current couplers. IEEE Trans. Energy Convers. 2014, 29, 224-231. [CrossRef]

18. Mohammadi, S.; Mirsalim, M. Double-sided permanent-magnet radial-flux adjust speed eddy-current couplings: Three-dimensional analytical modelling, static and transient study, and sensitivity analysis. IET Electr. Power Appl. 2013, 7, 665-679. [CrossRef]

19. Li, Z.; Wang, D.; Zheng, D.; Yu, L. Analytical modeling and analysis of magnetic field and torque for novel axial flux eddy current couplers with PM excitation. AIP Adv. 2017. [CrossRef]

20. Li, Y.; Lin, H.; Yang, H.; Fang, S.; Wang, H. Analytical analysis of a novel flux adjustable permanent magnet eddy-current coupling with a movable stator ring. IEEE Trans. Magn. 2018, 3, 1-4. [CrossRef]

21. Wang, J.; Zhu, J. A simple method for performance prediction of permanent magnet eddy current couplings using a new magnetic equivalent circuit model. IEEE Trans. Ind. Electron. 2018, 65, 2487-2495. [CrossRef]

22. Russell, R.L.; Norsworthy, K.H. Eddy currents and wall losses in screened-rotor induction motors. Proc. IEE Part A Power Eng. 1958, 105, 163-175. [CrossRef]

(C) 2019 by the authors. Licensee MDPI, Basel, Switzerland. This article is an open access article distributed under the terms and conditions of the Creative Commons Attribution (CC BY) license (http:/ / creativecommons.org/licenses/by/4.0/). 